\title{
Movement Data
}

4 Jed A. Long*, Trisalyn A. Nelson

5 Spatial Pattern Analysis \& Research Lab, Department of Geography, University of

6 Victoria, Victoria, BC, Canada

$7 \quad *$ corresponding author: jlong@uvic.ca

8

\section{Pre-print of published version.}

\section{Reference:}

Long, JA and TA Nelson. 2013. A review of quantitative methods for movement data. International Journal of Geographical Information Science. 27(2). 292-318.

\section{DOI:}

http://dx.doi.org/10.1080/13658816.2012.682578

\section{Disclaimer:}

The PDF document is a copy of the final version of this manuscript that was subsequently accepted by the journal for publication. The paper has been through peer review, but it has not been subject to any additional copy-editing or journal specific formatting (so will look different from the final version of record, which may be accessed following the DOI above depending on your access situation). 


\section{Abstract}

12 The collection, visualization, and analysis of movement data is at the forefront of

13 geographic information science research. Movement data are generally collected by

14 recording an object's spatial location (e.g., XY coordinates) at discrete time intervals.

15 Methods for extracting useful information, for example space-time patterns, from these

16 increasingly large and detailed datasets have lagged behind the technology for generating

17 them. In this article we review existing quantitative methods for analyzing movement

18 data. The objective of this article is to provide a synthesis of the existing literature on

19 quantitative analysis of movement data while identifying those techniques that have merit

20 with novel datasets. Seven classes of methods are identified: 1) time geography, 2) path

21 descriptors, 3) similarity indices, 4) pattern and cluster methods, 5) individual-group

22 dynamics, 6) spatial field methods, and 7) spatial range methods. Challenges routinely

23 faced in quantitative analysis of movement data include difficulties with handling space

24 and time attributes together, representing time in GIS, and using classic statistical testing

25 procedures with space-time movement data. Areas for future research include:

26 investigating equivalent distance comparisons in space and time, measuring interactions

27 between moving objects, development of predictive frameworks for movement data,

28 integrating movement data with existing geographic layers, and incorporating theory

29 from time geography into movement models. In conclusion, quantitative analysis of

30 movement data is an active research area with tremendous opportunity for new

31 developments and methods. 


\section{1 - Introduction}

33 The study of movement in geographic information science (GISci) has followed a

34 similar trajectory to the discipline of geography, whereby early work relied heavily on

35 qualitative methods. In the 1960's and 70's the discipline of geography experienced a

36 quantitative revolution whereby theory and methods were developed for explaining how

37 place and space could be modeled as quantitative entities. The quantitative revolution

38 produced developments in statistical methods designed specifically for spatial data, for

39 instance spatial autocorrelation measures (Cliff and Ord 1973). Only later in the

40 quantitative revolution did theoretical frameworks for quantitative analysis of movement

41 emerge; most notably Hägerstrand's (1970) time geography. As the quantitative

42 revolution in geography sputtered in the late 1970's (Johnston 1997) Hägerstrand's ideas

43 were primarily used as context for examining human behavior (e.g., Parkes and Thrift

44 1975, Pred 1981), rather than as an analytical toolkit for quantitative research. An

45 exception is the work of Lenntorp (1976) and Burns (1979), which represent seminal

46 pieces using time geography in quantitative analysis.

47 In the 1990's, triggered by the development of geographic information systems

48 (GIS), quantitative analysis again moved to the forefront of the geographic literature

49 (Sheppard 2001). The term geographic information science (GISci) was coined to refer

50 collectively to the science behind the collection, storage, representation, and analysis of

51 geographic datasets (Goodchild 1992). The term amalgamated those interested in the

52 study of geographic information including geographers, computer scientists, and

53 statisticians. As technologies for recording the paths of moving objects have evolved

54 (e.g., video, cell-phone, and GPS tracking) contemporary GIScientists have found new 
55 opportunities for quantitative analysis using time geography with GISci (e.g., Miller

56 1991, Kwan 1998). Other quantitative methods for analyzing movement have stemmed

57 from geography's strong legacy in spatial point pattern analysis (e.g., Gao et al. 2010), as

58 movement data are commonly represented by a sequence of points. Computational

59 geometry has played a leading role in recent advances in analyzing movement data (e.g.,

60 Laube et al. 2005). As well, methods for representing movement data using areal data

61 formats, for example polygons (Downs and Horner 2009) or fields (Downs 2010), remain

62 ongoing research areas. The study of movement is of interest in many applications

63 outside of GISci, for example wildlife ecology (Nathan et al. 2008), urban planning

64 (Drewe 2005), and military applications (Wells 1981). Further, the study of movement

65 has a long history in physics. Even Hägerstrand's time geography was strongly

66 influenced by the ideas of physicists from the early $20^{\text {th }}$ century (Rose 1977 , Hallin

67 1991). For example, the diagram of the space-time cone from time geography can be

68 clearly related to the past and future light-cones used in Einstein's relativity.

69 Movement is a complex process that operates through both space and time.

70 Representing the temporal dimension in geographic studies has presented a challenge for

71 GISci to move beyond static (map-based) representations of space (Chrisman 1998,

72 Laube et al. 2007). Despite notable advances at incorporating temporal dynamics in

73 GISci (e.g., Pultar et al. 2010), integrating the study of space and time remains at the

74 forefront of GISci research, as evidenced by the special symposium on space-time

75 integration in GISci at the 2011 annual meeting of the Association of American

76 Geographers. How to effectively integrate time into the quantitative analysis of

77 movement, specifically movement data stored in a GIS, is at the core of this review. 
The growth of spatial methods for quantitative analysis of movement data has

79 been facilitated by developments in movement databases that now provide efficient

80 methods for storing, indexing, and querying movement data (Güting and Schneider

81 2005). Despite the large body of existing literature on the topic of moving object

82 databases, it remains an active area of research as new tools (e.g., Güting et al. 2010a)

83 and applications (e.g., Jensen et al. 2010) continue to develop. Data visualization

84 methods have developed alongside these readily available movement databases; in GISci

85 this practice is termed geovisualization (Dykes et al. 2005). Given the sheer volume of

86 data often contained in movement databases, geovisualization can be a powerful tool for

87 identifying patterns in movement databases - a process referred to as visual analytics

88 (Thomas and Cook 2005). A complete treatment of either of these topics is beyond the

89 scope of this review, and we restrict the contents of this review to, as the title suggests,

90 those methods for analyzing movement data that are quantitative in nature. We would

91 point those interested in more information on movement databases to the comprehensive

92 book by Güting and Schneider (2005) and a recent special issue on data management for

93 mobile services (VLDB Journal, 20(5), Güting and Mamoulis 2011). For those interested

94 in more information on visual analytics for movement data we refer readers to Andrienko

95 and Andrienko (2007), and to the special issue from IJGIS entitled geospatial visual

96 analytics: focus on time (IJGIS, 24(10), Andrienko et al. 2010).

97 The objective of this review is to provide an unbiased evaluation of the usefulness

98 and shortcomings of existing quantitative methods for movement data, while highlighting

99 techniques that have particular merit with emerging movement datasets. Challenges to the

100 development and application of quantitative methods with movement data are identified 
101 in an attempt to locate avenues for future research. An outline of this article is as follows;

102 section 2 contains a brief introduction to the properties of movement data, and how

103 movement data is typically represented in a GIS. In section 3 we review the existing

104 literature on quantitative analysis of movement data separated into seven classes of

105 methods: 1) time geography, 2) path descriptors, 3) similarity indices, 4) pattern and

106 cluster methods, 5) individual-group dynamics, 6) spatial field methods, and 7) spatial

107 range methods. With section 4 we provide a discussion of the challenges routinely faced

108 in GISci when analyzing movement data and, what we feel are, some future directions for

109 quantitative movement analysis. Lastly, we close with some conclusions.

\section{2 - Movement Data}

111 Movement is a continuous process that operates in both the spatial and temporal

112 domains. Movement data are used to represent the continuous process of movement for

113 geographical analysis. Due to existing geospatial data collection and storage techniques,

114 movement data are most commonly represented as a collection of spatial point objects

115 with time stored as an attribute. A more formal definition of movement data is the

116 collection $\left\{M_{t}\right\}$ of $t=1 \ldots n$ ordered records each comprised of the triple $\langle I D, S, T\rangle$,

117 where $I D$ is a unique object identifier, $S$ are spatial coordinates, and $T$ a sequential (non-

118 duplicated) time-stamp (Hornsby and Egenhofer 2002). A number of terms are used

119 synonymously for movement data (see Table 1); here we use the term path to represent

120 the ordered sequence of records portraying individual/object movement, the term fix

121 when discussing a single record from a path, and the term movement database to describe

122 a collection of paths. The term movement data is used in broader contexts when 
123 discussing the study of movement, to refer generally to fixes, paths, and movement

124 databases.

125 <approximate location Table 1>

126 While movement data have historically been collected using a variety of

127 techniques, most current acquisition schemes use some form of wireless sensor (e.g.,

128 GPS, cellular phone records, radio telemetry). Calenge et al. (2009) identify two types of

129 sampling commonly employed in the collection of movement data - regular and

130 irregular. Regular paths are those where fixes are acquired at an even temporal interval,

131 for example recording one fix per minute. Irregular paths are those where fixes are

132 acquired at unequal temporal intervals, for example paths collected from cell phone call

133 records. The term granularity is used to refer to the resolution of a path (Hornsby and

134 Egenhofer 2002). Finer granularities are associated with frequent sampling intervals, and

135 provide a detailed representation of movement. Conversely, coarser granularities

136 correspond to sparse sampling and less-detailed representation of movement.

137 Technological developments now facilitate finer sampling intervals in movement paths

138 (e.g., 1 fix / second), and movement data can be used to represent a (near) continuous

139 movement path (Laube et al. 2007). However, these sensor-specific sampling designs

140 may not be suitable for all analysis questions, requiring the use of re-sampling (up- or

141 down-sampling) to fit a given research need (see Turchin 1998, and Hornsby and

142 Egenhofer 2002 for a more thorough discussion of changing granularity).

143 Spaccapietra et al. (2008) present an alternative view of movement data

144 granularity, defining a path as consisting of stops and moves separating a path into

145 periods of movement and stationary behavior. This conforms with the event-based model 
146 for movement data outlined by Stewart Hornsby and Cole (2007) which contrasts with

147 the coordinate-based representation of movement typically employed. An event based

148 model for movement data still allows for the detection of movement patterns, but with

149 focus placed on combinations or sequences of events that identify a specific behavior,

150 such as an exodus of objects out of a zone or region (Stewart Hornsby and Cole 2007).

151 Further, event based models allow for enriching movement data with the geographic

152 information associated with events, for instance if events are related to spatial regions the

153 attributes of each region.

154

1553 - Review of Methods

156 This section contains a review of quantitative analysis methods that exist within

157 seven areas of movement research; 1) time geography, 2) path descriptors, 3) path

158 similarity indices, 4) pattern and cluster methods, 5) individual-group dynamics, 6)

159 spatial field methods, and 7) spatial range methods. We emphasize techniques we feel

160 have particular merit for analysis with novel and emerging movement datasets.

\section{1 - Time Geography}

162 The concept of time geography was first presented in the 1960's and 1970's by

163 Torsten Hägerstrand at the Research Group for Process and System Analysis in Human

164 Geography at the University of Lund, Sweden (Lenntorp 1999). Time geography

165 (Hägerstrand 1970) represents a framework for investigating the constraints, such as an

166 object's maximum travel speed, on movement in both the spatial and temporal

167 dimensions. Hägerstrand expanded on the purely physical limitations of movement,

168 identifying three other types of constraints: capability, coupling, and authority 
169 constraints. Capability constraints limit the activities of the individual because of their

170 biological construction and abilities, for example the necessity to eat and sleep. Coupling

171 constraints represent specific locations in space-time an individual must visit that limit

172 movement possibilities. Authority constraints are opposite of coupling constraints,

173 locations in space time an individual cannot visit, for example a mall after it has closed.

174 Contemporaries expanded on Hägerstand's work providing both theoretical (Parkes and

175 Thrift 1975, Pred 1981) and applied (Lenntorp 1976, Burns 1979) extensions. Originally,

176 time geography was used solely to investigate the movement of humans, but has since

177 been reformulated for use with transportation networks (Miller 1991) and wildlife

178 ecology (Baer and Butler 2000).

179 Time geography uses volumes (Figure 1) capable of capturing the movement

180 limits of an object. A 3-D space (often termed cube, Kraak 2003, or aquarium, Kwan

181 2004), with two spatial axes representing geographic space and a third orthogonal axis for

182 time, is used to develop time geography volumes. The space-time cone (Figure 1a)

183 identifies the future movement possibilities of an object. A space-time prism (Figure 1b)

184 is used to quantify movement possibilities between known start and end locations. The

185 potential path area is the projection of the space-time prism onto geographic space

186 (Figure 1c), and is a purely spatial measurement of movement capability. A path is used

187 to portray the trajectory of movement through space-time. Bundling (Figure 1d) occurs

188 when multiple paths coincide in space and time, for example taking the same bus to

189 work. Typically, time geography is discussed qualitatively in terms of the aforementioned

190 volumes, but Miller (2005) has provided mathematical definitions for time geography

191 concepts that can be used in more rigorous quantitative analyses. 
195 has reached 'the end of it's beginning', suggesting that current and future research using

196 GIS and novel movement datasets will present new and exciting developments in time

197 geography. Examples include using time geography to investigate mobility data on a

198 network (Miller and Wu 2000), factoring in uncertainty (Neutens et al. 2007), field-based

199 time geography (Miller and Bridwell 2009, further discussed in S3.6), and the

200 development of a probabilistic time geography (Winter 2009, further discussed in S3.6).

201 Time geography represents a useful tool for quantitative analysis of movement as

202 it contains a framework for measuring space-time bounds on movement. Movement

203 models that fail to consider the constraints provided by space and time often result in

204 misleading conclusions (Long and Nelson 2012). Methods that explicitly consider time

205 geography principles, even unknowingly (e.g., Yu and Kim 2006), avoid such deceptions.

\section{2 - Path Descriptors}

207 Path descriptors are measurements of path characteristics, for example velocity, 208 acceleration, and turning azimuth. Typically path descriptors may be calculated at each

209 point in a movement dataset, and can be scaled appropriately to represent interval or

210 global averages. Dodge et al. (2008) categorize a number of path descriptors as primitive

211 parameters, primary derivatives, or secondary derivatives based on simple measurements

212 in space, time, and space-time (see Table 2). Ecologists routinely use simple path

213 descriptors in the study of wildlife movement (Turchin 1998). Measures of movement

214 tortuosity have also been developed for the study of wildlife, for example path entropy 
215 (Claussen et al. 1997), sinuosity (Benhamou 2004), and fractal dimension (Dicke and

216 Burrough 1988). Related to these are stochastic movement models (i.e., models where

217 fixes are obtained via random draws from distributions for movement displacement and

218 turning angle) such as Lévy flights (Viswanathan et al. 1996) and correlated random

219 walks (Kareiva and Shigesada 1983). When movement data are statistically fit to such

220 models, interpretation of model parameters can provide useful quantitative inference.

221 <approximate location Table 2>

\section{$222 \quad 3.3$ - Path Similarity Indices}

223 Path similarity indices are routinely used to quantify the level of similarity

224 between two movement trajectories. It is desirable for similarity indices to take the form

225 of a metric distance function, as metric functions are able to distinguish objects on an

226 interval scale of measurement (Sinha and Mark 2005). A metric distance function $(d)$ is

227 one that computes a generalized scalar distance between two objects while satisfying the

228 following four properties (Duda et al. 2001):

$229 \quad$ (i) Non-negativity: $d(x, y) \geq 0$;

230

(ii) Reflexivity (uniqueness): $d(x, y)=0$, iff $x=y$;

$231 \quad$ (iii) Symmetry: $d(x, y)=d(y, x)$;

232

233

(iv) Triangle Inequality: $d(x, z) \leq d(x, y)+d(y, z)$

235 implement a time-weighted distance metric where spatial proximity (Euclidean) is

236 weighted by its temporal duration. Sinha \& Mark (2005) also present a modified version

237 of the time-weighted distance metric for the situation where the two objects move over

238 different time intervals. Because the time-weighting is based on the duration an object

239 spends at a given spatial location, this index works best with movement data defined as a

240 series of stops and moves such as suggested by Spaccapietra et al. (2008). Yanagisawa et 
241 al. (2003) present an alternative Euclidean-based similarity index that focuses on the

242 shape of the movement path by normalizing the spatial coordinates of a path to a

243 common plane. Euclidean measurements in the normalized spatial plane are used to

244 identify similarly shaped movement paths. Euclidean distance is appropriate for

245 comparisons in the spatial or temporal domains. However, Euclidean measurements are

246 limited when data are represented with different scales (spatial and temporal). That is,

247 what is the temporal equivalent to a $1 \mathrm{~km}$ distance in space? Despite these limitations,

248 Euclidean distance similarity indices are frequently implemented by fixing either space or

249 time and considering Euclidean distance in the other dimension, such as the above

250 examples.

251 Other distance metrics may be more appropriate for assessing path similarities.

252 The Hausdorff distance is a shape comparison metric commonly used to evaluate the

253 similarity of two point sets (Huttenlocher et al. 1993), which has also been used to

254 measure the similarity of movement paths. Given two movement paths $M^{a}$ and $M^{b}$, the

255 Hausdorff distance is defined as:

$256 H\left(M^{a}, M^{b}\right)=\max \left(h\left(M^{a}, M^{b}\right), h\left(M^{b}, M^{a}\right)\right)$

257 with $h\left(M^{a}, M^{b}\right)=\max _{t \in T}\left(\min _{s \in S} d\left(M_{t}^{a}-M_{s}^{b}\right)\right)$

258 where $t$ and $s$ are used to index fixes from $M^{a}$ and $M^{b}$ respectively, and $d$ is a distance

259 operator (e.g., Euclidean). Not originally designed for movement data, the Hausdorff

260 distance performs poorly when analyzing movement paths as it fails to consider the

261 ordering of points (Zhang et al. 2006), and is sensitive to outliers and data noise (Shao et

262 al. 2010). As such, modified versions of the Hausdorff distance metric have been 
263 designed specifically for use with movement paths (e.g., Atev et al. 2006, Shao et al.

264 2010).

265 The Fréchet distance metric may be more appropriate as a path similarity index as

266 it was initially designed for comparing polygonal curves. Formally the Fréchet distance

267 for two movement paths $M^{a}$ and $M^{b}$ is defined as:

$268 \delta_{F}\left(M^{a}, M^{b}\right)=\inf _{\alpha, \beta} \max _{t \in[0,1]} d\left(M^{a}(\alpha(t)), M^{b}(\beta(s))\right) \quad$ [3]

269 Where $\alpha$ (resp. $\beta$ ) is an arbitrary continuous non-decreasing function from [0,1] onto

$270\left[t_{1} \ldots t_{n},\right]\left(\operatorname{resp} .\left[s_{1} \ldots s_{n},\right]\right)$ and $d$ is a distance operator (Alt and Godau 1995). In simple

271 terms, the Fréchet distance measures the maximum distance apart of two coinciding

272 movement paths. The Fréchet distance, is best conceptualized using the analogy of a

273 person walking their dog, where no backwards movement is allowed. In the dog walking

274 example, the Fréchet distance is the minimum length of the dog's leash. The discretized

275 form of the Fréchet distance metric (Eiter and Mannila 1994) is useful for its computation

276 with movement data collected by discrete fixes, as described in section 2. In applications

277 involving objects that move with the same temporal granularity this calculation is simply

278 the maximum distance in space between any pair of fixes taken at the same time.

279 However, when object movement is recorded at differing temporal granularities or

280 extents, the value of the Fréchet distance metric is through the use of the scaling

281 functions $(\alpha, \beta)$ to measure similarity.

282 Vlachos et al. (2002) use longest common subsequences (LCSS), a method taken

283 from time-series analysis, to identify similar movement paths. The LCSS is defined as the

284 number of consecutive fixes from two (or more) paths $\left(M^{a}, M^{b}, \ldots\right)$ that are within $d$

285 spatial and $\tau$ temporal units of each other. This method can be extended to paths that 
286 move at a distance, using mapping function $f(M)$ to translate $M^{b}$ onto a space equivalent

287 to $M^{a}$. LCSS is advantageous as it is able to address issues relating movement paths taken

288 at different temporal granularities and/or extents. LCSS is efficient even with paths that

289 contain a significant amount of data noise. When outlying fixes are likely to influence the

290 calculation of other similarity indices LCSS is advantageous as it is insensitive to

291 extreme outliers. The disadvantage of the LCSS method is that it relies on the subjective

292 definition of thresholds $-d$ and $\tau$, and it fails the triangle inequality test (iv. above), and is

293 therefore not a metric distance function.

294 Similarity indices have also been extended to objects moving along a network.

295 For example, Hwang et al. (2005) calculate similarity using points-of-interest, such as

296 major intersections. Movement paths are considered similar if they pass through the same

297 points-of-interest in the same order. This index is not a metric distance function, but

298 moves away from Euclidean based measurements which are inappropriate in a network

299 scenario.

300 Recently, a new similarity method has been proposed by Dodge et al. (2012).

301 Here, a movement path is separated into segments where specific movement parameter

302 patterns (and derivatives of) are observed. In their example, velocity is the parameter of

303 interest, and the metrics deviation from the mean and sinuosity are used to define

304 movement parameter classes. For example, the letters A-D could be used to denote 4

305 unique movement parameter classes, and a path could then be represented as the

306 sequence [ACBCACBDBDA]. To assess the similarity of two paths, a modified version

307 of the edit distance (a string matching algorithm) is computed on the movement

308 parameter class sequences. This method measures similarity in the selected movement 
309 parameters, rather than in the space-time geometry of the movement paths. As such, it

310 may be more appropriate when similarity in various parameters, rather than space-time

311 geometry is specifically of interest, for instance, in the study of hurricane path dynamics,

312 as demonstrated by Dodge et al. (2012).

313 When objects interactively move with each other at a distance, they often exhibit

314 correlated movement. Typically, similarity indices may identify such correlated

315 movements by mapping the spatial coordinates of one path onto the spatial plane

316 equivalent to the other. Alternatively, Shirabe (2006) presents a method for computing

317 the correlation coefficient between two movement paths, each represented as a vector

318 time-series. Consider a path $M$ with $t=1 \ldots n$ fixes, then for $t=2 \ldots n, V=\left[M_{t}-M_{t-1}\right]=$

$319\left[v_{t}\right]$, is a vector time series of $M$. Given two two movement paths $\left(M^{v}, M^{w}\right)$ represented as

320 vector time-series $\boldsymbol{V}$ and $\boldsymbol{W}$, the correlation coefficient is defined as:

$$
r(\boldsymbol{V}, \boldsymbol{W})=\frac{\sum_{t=1}^{n-1}\left(\boldsymbol{v}_{t}-\overline{\boldsymbol{v}}\right) \cdot\left(\boldsymbol{w}_{t}-\overline{\boldsymbol{w}}\right)}{\sqrt{\sum_{t=1}^{n-1}\left|\boldsymbol{v}_{t}-\overline{\boldsymbol{v}}\right|^{2}} \sqrt{\sum_{t=1}^{n-1}\left|\boldsymbol{w}_{t}-\overline{\boldsymbol{w}}\right|^{2}}}
$$

322 Where $\overline{\boldsymbol{v}}=\frac{1}{n-1} \sum_{t=1}^{n-1} \boldsymbol{v}_{t}($ resp. $\bar{w})$ are mean coordinate vectors of $(\boldsymbol{V}, \boldsymbol{W})$. Note that a

323 movement path of $n$ fixes is comprised of $n$-1 movement vectors, this distinction we keep

324 for consistency with other methods. The numerator in [4] is the covariance, which

325 indicates how the two motions deviate together from their respective means (Shirabe

326 2006). Geometrically, the dot product in the numerator is the product of vector lengths

327 multiplied by the cosine of the angle between them, which can be interpreted as the

328 similarity. The correlation index ranges from -1 to 1 , identifying both negatively and

329 positively correlated movements. Important to note is that this correlation coefficient 
330 relies on each movement's deviation from the respective mean, not the raw values of

331 each observed movement. Relating correlations to a global mean can be advantageous in

332 cases where two movements are correlated, but do not move in the same direction. The

333 first drawback of the formulation in [4] is that we are unable to disentangle the effects of

334 correlation in azimuth vs. magnitude of movements. A metric decomposed into each of

335 these components would be advantageous in situations where such distinctions are

336 necessary. A second drawback of equation [4] is that it requires that the fixes from each

337 movement path be taken simultaneously in order to be valid, which is not always

338 realistic. However, Shirabe (2006) does present an extension for modifying [4] to

339 measure movement path correlations at a temporal lag.

$340 \quad 3.4$ - Pattern and Cluster Methods

341 Many applications are interested in identifying broad spatial-temporal patterns

342 from large movement databases (Benkert et al. 2007, Palma et al. 2008, Verhein and

343 Chawla 2008). For example, in the study of tourist behavior, often the goal is to identify

344 places of interest that are frequently visited (e.g., Ahas et al. 2007). Alternatively,

345 studying commuter patterns typically involves the identification of intersections and

346 routes being used by multiple individuals (Verhein and Chawla 2006). In these situations,

347 pattern and cluster methods are employed to identify similar movement behaviors or 348 places of interest.

349 Early work on indexing and querying movement databases coming from the

350 computer and database science literature (e.g., Güting et al. 2000, Pfoser et al. 2000) has

351 been essential to the development of pattern and cluster methods. For instance, many

352 methods for identifying patterns and clusters in large movement databases implement a 
353 simple spatial or temporal query (Erwig et al. 1999). Alternatively, pattern or cluster

354 methods may implement one of the aforementioned path similarity indices and perform

355 pair-wise similarity computations over all permutations of stored movement paths. Paths

356 identified as similar based on a query or similarity index may convey some movement

357 pattern, or belong to the same cluster. The use of the term 'cluster' comes from methods

358 for statistical analysis of spatial point patterns (Diggle 2003), as many approaches used in

359 point pattern analysis have been adopted for movement data. For example, both Gao et al.

360 (2010) and Güting et al. (2010b) describe methods for performing $k$-nearest neighbor

361 queries in movement databases.

362 For the most part, the identification of patterns and clusters in large movement

363 databases focus on one of space, time, or space-time. Methods that identify spatial

364 clusters look at space first and time second, if at all (e.g., Benkert et al. 2007). The

365 simplest methods for detecting spatial clusters in movement databases generally require

366 that fixes from individual paths be represented as spatial points. Other spatial methods

367 look to define regions of interest (static or dynamic) and identify times at which

368 movement fixes are clustered in these spaces (Giannotti et al. 2007). Alternatively,

369 temporal clusters look at time first and space second, (e.g., D'Auria et al. 2005, Nanni and

370 Pedreschi 2006). Temporal clustering is enhanced (Palma et al. 2008) when movement

371 paths are represented by a sequence of stops (representing activities) and moves

372 (Spaccapietra et al. 2008).

373 Space-time approaches to identifying patterns and clusters strive to consider space

374 and time simultaneously. This is difficult, as previously mentioned, due to scaling

375 differences between space and time. Most space-time approaches fail to properly scale 
376 space and time and degenerate to spatial clustering methods linked through time (e.g.,

377 Kalnis et al. 2005). Such methods routinely consider the following problem: given $p$

378 mobile objects, $M^{i}, i=1 \ldots p$. Each $M^{i}$ consists of $n$ fixes taken at coinciding times $t=(1$,

$379 \ldots n)$. A set of $\alpha(1 \leq \alpha \leq p)$ spatial clusters are identified at each time $t$ (for example with

380 multivariate clustering) using the spatial $(x, y)$ coordinates of $M^{i}(t)$. In one example,

381 Shoshany et al. (2007) link clusters through time using linear programming. In their

382 example, moving objects $M^{i}$ can switch between clusters, but all $M^{i}$ must belong to a

383 cluster, as well clusters can emerge or disappear over time. The appeal of this approach is

384 that linear programming, frequently used in optimization research, can identify flows and

385 trends in movement data clusters.

386 Spatial-temporal association rules (STAR) learning represents an algorithm-based

387 method for discovering spatial-temporal patterns in movement databases (Verhein and

388 Chawla 2006, 2008). The patterns found by STAR methods are able to identify sources,

389 sinks, and thoroughfares in large mobility databases. Verhein and Chawla (2008)

390 demonstrate a STAR-miner software that implements their algorithm, and apply it to a

391 caribou dataset. STAR patterns rely on pre-determined spatial units (termed regions) over

392 which the algorithm is run. Unfortunately, the use of explicit spatial regions in their

393 derivation means that STAR are especially sensitive to changes in the definition of

394 regions (known as the modifiable areal unit problem - Openshaw 1984).

395 Pattern and cluster methods for movement data have also drawn on existing

396 methods from other applications. Shoval and Isaacson (2007) propose sequence

397 alignment methods, originally used to analyze DNA, as a way to identify patterns in

398 human travel behavior. With movement data, sequence alignment methods are able to 
399 identify groups of objects that follow a similar sequence of events (e.g., using an event

400 based movement data representation, as in Stewart Hornsby and Cole 2007). Shoval and

401 Isaacson (2007) apply sequence alignment methods to tourist movement data and

402 conclude that sequence alignment methods have potential for identifying patterns of

403 spatial behavior in large movement databases. In another example, Eagle and Pentland

404 (2009) introduce a method for discovering eigenbehaviors in movement databases.

405 Eigenbehaviors represent trends or routines in individual movement data. Principle

406 component analysis is used to identify the eigenbehaviors of each person in their dataset.

407 In their example using the movements of people's daily routines, three trends emerge:

408 workday, weekend, and other behaviors. Increasingly complex questions could be

409 addressed using the eigenbehavior method.

\section{$410 \quad 3.5$ - Individual-Group Dynamics}

411 The term individual-group dynamics is used to classify a suite of methods that

412 focus on individual object movement within the context of a larger group. This differs

413 fundamentally from methods for identifying patterns and clusters in movement databases.

414 Most current methods for investigating individual-group dynamics rely on computational

415 algorithms capable of searching movement databases for specific, pre-defined patterns.

416 These algorithms are often computationally demanding and inefficient (Gudmundsson et

417 al. 2007), and thus primarily used only in small, case-study examples.

418 Laube et al. $(2004,2005)$ provide the most comprehensive examination of

419 individual group-dynamics. Their concept of relative motion (REMO) can be used to

420 detect specific patterns (constancy, concurrence, and trend-setters) in groups of moving

421 objects. Constancy represents when an object moves in the same direction for a number 
422 of consecutive fixes. An episode of concurrence occurs when multiple moving objects

423 move in the same direction at the same time. Trend-setters are objects that move in a

424 given direction ahead of a concurrence episode by a group of objects. Trend-setting is

425 identified as the most interesting property, and examined in more detail using the sport of

426 soccer as an example. Players that exhibit trend-setting behavior are able to better

427 anticipate the movement of play. Their concept of trend-setting has been further

428 developed for identifying leaders and followers in groups of moving objects, which is

429 potentially useful for the analysis of wildlife movement data (Andersson et al. 2008).

430 Laube et al. (2005)'s REMO method uses only movement azimuths to determine relative

431 motion. All other movement attributes, such as speed or distance, are ignored in their

432 derivation. Thus, REMO is useful only in situations where a group of objects move with

433 similar speeds and are contained in a relatable geographic space, such as the soccer

434 example. Another disadvantage is that the REMO method relies on the definition of

435 azimuthal breakpoints to define when objects are moving in a similar direction (e.g., East

436 is between $45^{\circ}$ and $135^{\circ}$ ). Due to their discreteness, these breakpoints can lead to

437 misleading interpretations, for example when objects move in similar directions on either

438 side of a breakpoint. Alternatively, Noyon et al. (2007) evaluate the relative movement of

439 objects from the point-of-view of an observer within the system. Using changes in

440 relative inter-object distance and velocity, Noyon et al. (2007) identify relative behavior,

441 for example collision avoidance. Furthermore, Noyon et al. (2007) suggest that such

442 relative movement behavior also include other regions-of-interest such as lines and

443 polygons, which they include in their derivation. 
Another problem routinely encountered in the study of movement is the detection

445 of flocks and convoys (e.g., groups of individuals that move as a cohesive unit). A flock

446 (see Figure 2a) is defined as a group of at least $m$ moving objects $(M)$ contained within a

447 circle of radius $r$ over a minimum time interval - $\tau$ (Gudmundsson and van Kreveld 2006,

448 Benkert et al. 2008). Alternatively, a convoy (see Figure 2b) is defined as a group of at

449 least $m$ moving objects $(M)$ that are density connected at a distance $d$ over a minimum

450 time interval - $\tau$ (Jeung et al. 2008). Density connected implies that there exists a

451 sequence of segments connecting all points in the convoy, each segment with length $\leq d$.

452 This definition of convoy relaxes the circular requirement of flocks affording flexibility

453 in the shape and extent of convoys that can be identified, for example Canada geese

454 forming their characteristic V-shape. Methods that look at flock/convoy behavior have

455 obvious usefulness in the study of wildlife herds, but also in monitoring crowd dynamics

456 at large events (Benkert et al. 2008). Like space-time clustering, methods describing

457 flocks or convoys build upon Hägerstrand's concept of bundling, identifying areas where

458 objects move coincidentally in space-time. The fundamental difference between the

459 identification of flocks or convoys and space-time cluster methods is that the definition of

460 a flock or convoy explicitly considers the individual in relation to the group in its

461 definition. That is, focus is placed on membership to a given group, with explicit

462 consideration of minimum requirements for flock or convoy behavior (e.g., the

463 parameters $m$ and $\tau$ ). Space-time cluster methods focus more on identifying broader

464 patterns, typically from large movement databases, and generally rely on pair-wise

465 comparisons of individual movement paths.

466 <approximate location Figure 2> 
Recently, free space diagrams have been proposed for identifying single-file

468 motion in movement databases (Buchin et al. 2010). To conceptualize a free space

469 diagram consider two movement paths $\left(M^{a}\right.$ and $\left.M^{b}\right)$, over the time intervals $m$ and $n$

470 respectively, where the trajectory between fixes is given by some linear or other model

471 (e.g., Tremblay et al. 2006). The functions $\varphi_{a}$ and $\varphi_{b}$ give the position of the objects $a$ and

$472 \mathrm{~b}$ at time $t$. The free space diagram for $a$ and $b$ (following Buchin et al. 2010) is given by:

$473 \quad F_{\delta}\left(M^{a}, M^{b}\right)=\left\{\left(t^{a}, t^{b}\right) \in[1, n] \times[1, m]:\left|\varphi_{a}\left(t^{a}\right), \varphi_{b}\left(t^{b}\right)\right| \leq \delta\right\}$

474 which defines the set of all points in $\varphi_{a}$ and $\varphi_{b}$ that have a Euclidean distance below some

475 threshold $-\delta$. The map of $F_{\delta}$ describes a two dimensional space where the axes

476 correspond to the two paths, and the free space is defined as anywhere along the paths

477 where the distance between the two paths is below the threshold $\delta$. Buchin et al. (2010)

478 demonstrate a method for interpreting free-space diagrams capable of identifying single-

479 file movement patterns in groups of moving objects. A criticism of this method is that it

480 relies on a subjectively defined threshold $-\delta$, to constrain the single-file movement

481 process. Single-file motion has intuitive meaning, but is especially difficult to

482 conceptualize geometrically. Methods that use Euclidean geometry to measure the spatial

483 separation between leaders and followers (e.g., Andersson et al. 2008) are inadequate for

484 identifying single-file movement warranting the free-space diagram approach.

\section{$485 \quad 3.6$ - Spatial Field Methods}

486 Often it is of interest to represent a movement path (or many movement paths) as

487 a spatial field in order to identify areas in space (or space-time) that are more or less

488 frequently visited. Field based representations are especially useful for visualizing large

489 quantities of movement data when maps become cluttered. As many other spatial datasets 
490 are stored as raster fields, a field-based representation of movement allows quantitative

491 map comparisons to be performed in a GIS.

492 Most methods for representing movement data as spatial fields have evolved from

493 those used to analyze spatial point patterns. When spatial point pattern methods are

494 employed the temporal component of movement fixes is ignored. Spatial point pattern

495 methods can be separated into quadrat or density based methods (Diggle 2003). The

496 simplest quadrat methods involve subdividing a study area into a regular grid and

497 determining point densities within each cell (e.g., Dykes and Mountain 2003,

498 Hadjieleftheriou et al. 2003). Cells with high point densities indicate spatial locations of

499 high use. Hengl (2008) proposes a quadrat based space-time density measure based on

500 distance and velocity within each cell [6].

$501 \quad D_{x y t}(j)=\frac{\hat{d}_{j}}{\hat{v}_{j}} \quad$ [6]

502 Here $D_{x y t}(j)$ is the space-time density at cell $j, \hat{d}_{j}$ is the length of the movement path

503 within cell $j$, and $\hat{v}_{j}$ is the average velocity of movement within cell $j$. For a single

504 moving object the space-time density is simply interpreted as the duration of time the

505 object spends within each cell. If calculated for a movement database of many objects,

506 areas with higher space-time densities represent those where more objects spend more

507 time, the opposite with low values (Hengl et al. 2008). This approach has been extended

508 for three-dimensional visualization, where density is related to the lengths of multiple

509 paths in 3-D voxels defined by two spatial dimensions and a temporal dimension

510 (Demšar and Virrantaus 2010). Voxel densities are visualized in a space-time cube

511 (aquarium), and can be used for exploratory analysis of large movement databases. 
512 Density based methods in spatial point pattern analysis stem from bivariate

513 probability models, where movement fixes represent sampled locations from a two-

514 dimensional probability density function (Silverman 1986). In the analysis of wildlife,

515 density based models are frequently used to generate estimates of animal space use (also

516 discussed in S3.7). Worton (1989) first applied kernel density estimation (KDE) to

517 wildlife movement data to derive such a surface, termed a utilization distribution

518 (Jennrich and Turner 1969). In movement applications, KDE can be interpreted as the

519 intensity of space use based upon a collection of fixes. Calculation of KDE requires

520 selection of a kernel shape and bandwidth parameter, with no consensus on the best way

521 to do so (Hemson et al. 2005, Kie et al. 2010). Alternatively, Downs (2010) has proposed

522 time geography's potential path area (see Figure 1) to replace the kernel shape and

523 bandwidth parameter, representing a novel approach for integrating temporal constraints

524 into KDE analysis. Downs (2010) replaces the traditional kernel function with one based

525 on the potential path area (termed geo-ellipse $-\boldsymbol{G}$ ) from time geography [7].

$526 \quad \hat{f}_{t}(x)=\frac{1}{(n-1)\left[\left(t_{E}-t_{S}\right) v\right]^{2}} \sum_{i=1}^{n-1} \mathrm{G}\left(\frac{\left\|x-M_{i}\right\|+\left\|M_{j}-x\right\|}{\left(t_{j}-t_{i}\right) v}\right)$ [7]

527 The numerator in this function sums the distance between a given point $x$ and the object's

528 locations $(M)$ at times $i$ and $j$. The denominator is the maximum distance the object could

529 have travelled in that time interval given its maximum velocity $-v$. Others have seen the

530 need to move away from continuous representations of space, and have developed KDE

531 for networks (Borruso 2008, Okabe et al. 2009). Such analysis is more appropriate for

532 depicting the movement of urban travelers as their movement is restricted to travel

533 networks of roads, paths, and sidewalks. 
535 continuous spatial field. Horne et al. (2007) use Brownian bridges to model wildlife

536 movement as a continuous probability surface. Between two consecutive mobility points

537 the probability an object is at a given location at time $t$ is defined using a bivariate normal

538 probability density function. More recently, probabilistic time geography has been

539 proposed (Winter 2009), where a similar probability surface is based on discrete random

540 walks in a cellular automata environment. Winter \& Yin (2010) extend on the ideas of

541 Winter (2009) to include directed movements. Random walks are used to derive a

542 probability surface which explicitly considers the time geographic constraints on object

543 movement, using a similarly defined bivariate normal probability surface. Both Winter \&

544 Yin (2010) and Horne et al. (2007) discuss the fact that determining movement

545 probabilities based on random walks is limited when objects do not move randomly.

546 Future work looking at probabilistic movement using other movement models (e.g.,

547 correlated random walks or on a network) is thus warranted for moving objects that can

548 be modeled this way. Alternatively, Miller \& Bridwell (2009) propose a field-based time

549 geography. Field-based time geography uses movement cost surfaces in the calculation of

550 time geography volumes. Movement possibilities are evaluated in a similar manner to

551 Winter and Yin (2010) but based on an underlying movement cost surface (e.g., as in

552 least-cost path analysis in GIS, Douglas 1994). This approach is advantageous in that it

553 directly considers underlying variables impacting movement, however is limited in that

554 an accurate cost surface must be derived.

555 Brillinger et al. $(2001,2004)$ provide a unique approach for discovering patterns

556 in movement data. Stochastic differential equations are used to model movement as a 
557 Markov process. The drift term in the stochastic movement model can be interpreted as a

558 spatial velocity field and used for exploratory analysis. The spatial velocity field

559 represents a potential function, whereby points of attraction and repulsion can be

560 identified. Methods for statistical inference (e.g., jackknifing) can be used to identify

561 statistically significant movement patterns within this velocity field (Brillinger et al.

562 2002). Brillinger (2007) further applies this approach for analyzing the flow of play in

563 soccer, where the spatial velocity field for ball movement is used to investigate a team's

564 attack formation.

565 3.7-Spatial Range Methods

566 Spatial range can be broadly defined as the area (generally represented as a

567 polygon) containing an object's movement. Measures of spatial range can be useful for

568 examining object mobility and space use. Aspatial metrics, such as net displacement

569 (Turchin 1998), provide no information on the spatial distribution of movement, simply

570 measuring distance, thus spatial measurements are warranted. Furthermore, researchers

571 are often interested in intersections and/or differences in movement ranges (e.g., Righton

572 and Mills 2006). In such cases it is advantageous to represent point/line movement data

573 in an areal format (e.g., as a polygon).

574 The practice of representing movement data using spatial polygons has been

575 developed primarily by wildlife ecologists for studying wildlife home ranges (Burt 1943),

576 however, the concept of home range has also been applied to other subjects (e.g.,

577 children, Andrews 1973). Spatial range methods typically rely on the geometric

578 properties of movement data, for example the calculation of the minimum convex

579 polygon, a common measure of wildlife home range (Laver and Kelly 2008). Other 
580 geometric methods include harmonic mean (Dixon and Chapman 1980), Voronoi

581 polygons (Casaer et al. 1999), and characteristic hull (Downs and Horner 2009). It is also

582 common to extract spatial range polygons from spatial field representations of movement

583 (e.g., those from S3.6) by extracting polygon contours based on density. For example,

584 with KDE a 95\% volume contour is frequently used to delineate wildlife home range,

585 while a 50\% volume contour is used to delineate core habitat areas (Laver and Kelly

586 2008). These spatial range methods ignore temporal information stored in movement data

587 and are likely to contain areas never visited by the object (commission error), and miss

588 actually visited locations (omission error) (Sanderson 1966).

589 Time geography volumes may also be used for generating spatial range estimates.

590 Long \& Nelson (2012) propose a spatial range method for wildlife movement data based

591 on time geography's potential path area (Figure 1c). This method is capable of

592 identifying omission and commission errors in other spatial range methods (Long and

593 Nelson 2012). Such time geographic analysis is commonly used to study accessibility in

594 the context of human movement (Kwan 1998). The value of the potential path area as a

595 spatial range method is that it explicitly considers the temporal sequencing of movement

596 data in a time geography context. Spatial range methods that consider the temporal

597 component of movement data are advantageous over purely spatial methods (such as

598 convex polygons) as they consider movement data as a sequence of spatial points taken

599 through time, rather than as an arbitrary collection of spatial points.

600

601 4-Discussion

$602 \quad 4.1$ - Time 
The first and foremost challenge to the quantitative analysis of movement data is

604 how to effectively characterize time. Despite having well-developed theory and tools for

605 analyzing space, geographers and the GISci community have historically struggled with

606 the temporal dimension (Peuquet 1994). Time is a single, continuous dimension that can

607 be portrayed as either monotonically linear or cyclical (Frank 1998). If time is portrayed

608 as linear, objects are not capable of re-visiting instances in time. If time is portrayed as

609 cyclical, the beginning of a new cycle infers that time is reset to some initial state, thus

610 revisiting is facilitated. For example, consider research on human daily routines; within

611 each day time is treated linearly, but is reset at the beginning of each day signifying the

612 start of a new cycle. Movement data collected over long periods may contain both linear

613 and cyclical temporal patterns, confounding representation and analysis.

614 Theoretical constructs for including time in GIS have long been discussed

615 (Langran and Chrisman 1988, Peuquet 1994) but remain challenging. Some spatial

616 datasets are easily represented at discrete time intervals in a GIS as different layers, for

617 example land cover data in different years. This representation allows for vertical

618 analysis through time using relatively simple map algebra (Mennis et al. 2005). Vertical

619 analysis through time is not straightforward with movement data, as objects move in both

620 space and time and cannot be explicitly linked through the spatial dimension. Others have

621 suggested the notion that geography's fetish for the static (Raper 2002) may lie at the root

622 of the time problem. In practice, researchers have begun to use a 3-D aquarium (drawing

623 on Hägerstrand's ideas) for representing time in GIS, however this is principally a

624 visualization tool (e.g., Kraak 2003, Andrienko and Andrienko 2007, Shaw et al. 2008).

625 Dynamic views (i.e., animations) may overcome the drawbacks of static portrayals of 
626 movement, allowing more fluid representations of velocity and acceleration properties

627 (Andrienko et al. 2005). However, dynamic views are also visual-based, and lack

628 potential for developing quantitative analyses.

629 The challenge has been finding appropriate ways to simultaneously represent the

630 different scales of measurement for temporal and spatial attributes associated with

631 movement. Consider that it is common to use measurements of time and space

632 interchangeably in queries associated with movement from everyday life, for example if

633 you were asked the question: how far is it from here to the grocery store? You might

634 answer with "about 2 kilometers" or alternatively with "about a 5 minute drive". Here, a

635 question of spatial distance associated with movement can be equivalently answered

636 using a spatial measurement $(2 \mathrm{~km})$ or temporal measurement ( 5 minutes). This has led to

637 alternative conceptualizations of movement where space and time can be represented

638 using relationships that can scale from spatial to temporal measurements, and vice-versa

639 (Parkes and Thrift 1975). For example, travel can be considered as the consumption of

640 physical distance through time (Forer 1998). However in the previous scenario, you may

641 have also answered with "about a 5 minute drive, depending on traffic". Alternatively,

642 one might add that it depends on mode of transport (e.g., whether you walk or drive).

643 This alternative view demonstrates the non-linear and dynamic relationship that exists

644 between space and time which confounds the direct exchange of measurements of space

645 and time (Forer 1998). With movement data, time is often stored alongside spatial

646 attributes (e.g., $<x, y, t>$ ), which naturally lends itself to Euclidean-type measurements in

647 the space-time aquarium. However, as demonstrated, time is poorly represented by such

648 direct physical measurements, because time cannot be represented as a linear function of 
649 space. As there is still no consensus on the best way to represent time with movement

650 data, research on how to effectively characterize space and time in movement data

651 continues to require development.

652 Distance in space is easily computed using Euclidean (or other, such as network)

653 measurements. Differences in time are generally measured using clock times. The

654 conceptualization of a single space-time proximity measure remains one of the biggest

655 hurdles with quantitative analysis of movement data. Moving forward it is imperative to

656 go beyond simple Euclidean based measures, as time and space do not operate on equal

657 scales (Peuquet 2002). The Fréchet distance (Alt and Godau 1995) is an example of a

658 novel method for comparing the similarity of two movement paths that may prove useful

659 in future analyses. Nearest neighbor computations (e.g., Gao et al. 2010), most useful

660 with movement data stored as points, may also provide avenues for exploration.

661 Normalizing different data scales, common to other branches of quantitative analysis

662 such as multivariate cluster analysis (Duda et al. 2001), may be useful for comparing

663 movement processes across scales and relates to work using fractals for describing

664 movement datasets (Dicke and Burrough 1988). Normalization, however, may mask

665 scale specific patterns, and should be done with caution only when scale specific

666 behavior is less-important. Fundamentally, space and time have different dimensions and 667 require special consideration when analyzed together.

670 With any spatial analysis the selection of analysis level (scale) will influence the 671 outcome of quantitative measures and the resulting inferences and conclusions (Dungan 
672 et al. 2002). The study of scale and its impacts in spatial analysis remains a key topic in

673 geographic studies. In the analysis of movement data Laube et al. (2007) identify four

674 levels of analysis: instantaneous, interval, episodal, and global (Figure 3). The

675 instantaneous ("local") level represents measures computed at any point along a

676 movement path. Interval ("focal") level analysis takes the form of a moving temporal

677 window, but may also use a moving spatial window. Episodal (“zonal”) level analysis

678 looks at specific partitions of movement data, often related to some known event. Most

679 common is global level analysis, where a movement dataset is represented as a complete

680 path, from beginning to end, as a single entity. While some methods are specifically

681 designed for a given level of analysis others can be applied to various levels. Methods

682 that can be applied at different analysis levels may not scale from one level to the next,

683 meaning results at a lower level may not sum to the global result, as is the case with some

684 spatially local statistics (termed LISA - Anselin 1995).

685 <approximate location Figure 3>

686 Quantitative methods are also sensitive to changes in the temporal granularity at

687 which movement data is represented (Laube and Purves 2011). Methods for changing

688 granularity can be used when process scale is explicitly known, however this is rarely the

689 case. When movement data are over-sampled (i.e., too fine a granularity) data noise can

690 mask broader-scale process signals. When movement data are under-sampled (i.e., too

691 coarse a granularity) important movement events are missed, leading to incorrect

692 parameter estimates. Some ecologists have suggested that movement data should not be

693 sampled at even time intervals, but rather as a sequence of moves or steps relating to

694 individual behavior (Wiens et al. 1993, Turchin 1998). This aligns with the view of 
695 Spaccapietra et al. (2008) that human movement data are best represented as a series of

696 stops (representing activities, as in the event-based model of Stewart Hornsby and Cole

697 2007) and moves. However, many developed methods tend to perform better when

698 implemented with regularly sampled movement data (e.g., Downs et al. 2012). As the

699 toolbox of methods for the quantitative of analysis of movement grows, it will be

700 important to identify at what analysis level(s) and over which temporal granularities

701 various methods are appropriate.

702 As previously identified, and following from Laube et al. (2007) and Laube and

703 Purves (2011), there are two fundamental issues of scale associated with movement

704 analysis, that is, analysis level and temporal granularity. Laube and Purves (2011)

705 suggest a third issue of scale may also exist, in that many approaches for movement

706 analysis are tested only on small, idealized datasets, and do not perform as expected when

707 carried out on larger, real-life datasets. As a result, many existing methods cannot be

708 readily implemented in practical scenarios with large volumes of movement data. We

709 take an alternative view on this issue. Testing of methods with smaller, idealized datasets

710 limits the scope of movement analysis to realistic and manageable problem sets, which

711 are in turn appropriate with subsets of a larger movement database. For example, the

712 detection of trend-setters (Laube et al. 2005) is only useful if there is some expectation

713 about where, if observed, this pattern is meaningful. In applied research, one should be

714 able to identify specific scenarios, within a larger movement database, where a given

715 technique is appropriate. Once these specific scenarios are identified, for example using

716 spatial-temporal queries, apply the technique of interest on this subset of the movement

717 database. The result is a multi-tiered analysis, where a specified technique is only 
718 performed on smaller, appropriate subsets of the data. The goal being to break down

719 larger movement datasets into pieces resembling the idealized scenarios upon which

720 various techniques are useful.

\section{$721 \quad 4.3$ - Statistical Significance}

722 Often, it is desirable to examine quantitative problems using a statistical lens, that

723 is, to determine if some pattern is different than an expectation. For those less familiar

724 with statistical inference in GISci, we point the reader to the text by O'Sullivan and

725 Unwin (2010), which provides an introduction to these concepts. Spatial statistics often

726 rely on the concept of complete spatial randomness (CSR) as an a priori assumption for

727 assessing the statistical significance of observed spatial patterns (Cressie 1993). With

728 some types of spatial statistics (e.g., join counts, Cliff and Ord 1981) the distributions for

729 computing statistical tests are analytically derived. With other statistics, specifically most

730 spatially local measures, simulation procedures are used to generate test distributions,

731 making these statistics primarily exploratory (Boots 2002).

732 Random walks have been suggested as being to movement data what CSR is to

733 spatial data (Winter and Yin 2010). Two key methodological developments have

734 included random movement in their derivation: Brownian bridge home ranges (Horne et

735 al. 2007) and probabilistic time geography (Winter and Yin 2010). However, these two

736 examples represent essentially the same problem: defining a probability surface for

737 movement between two known locations in space-time. Authors of both methods concede

738 that random movement is inappropriate for modeling objects that move non-randomly,

739 but contend that it represents a necessary starting point. 
740 The development of space-time statistics for movement is still in its infancy and

741 lacks clear direction for future research. Some have taken alternative views on this

742 problem, for example treating movement data as a bivariate time series using spatial

743 coordinates as dependent variables (e.g., Jonsen et al. 2003). Others have looked at

744 geographic space first, often ignoring the temporal component altogether (e.g., Casaer et

745 al. 1999). Both approaches are limited as they do not consider movement as a dynamic

746 process that is a function of both space and time. To adequately address the process of

747 movement, novel statistical techniques must consider space and time simultaneously in

748 their derivation. This will be challenging however, as inferential statistics are ill-suited to

749 the multidimensional complexity of movement (Holly 1978).

$751 \quad 4.4$ - Emerging Trends in Quantitative Movement Analysis

752 Technological advances now facilitate real-time capture and analysis of

753 movement data on both wildlife and humans. In wildlife applications, real-time data

754 acquisition is providing opportunities for conservation and wildlife management. Dettki

755 et al. (2004) implemented a real-time tracking system for moose in Sweden, where data

756 on moose movements could be used to initiate the start-up and shut-down of forestry

757 operations in seasonal moose ranges. This idea relates directly to recent work identifying

758 the importance of timing in time geographic measures of space-time accessibility

759 (Neutens et al. 2010, Delafontaine et al. 2011a). As the interface between wildlife and

760 humans narrows, other potential applications exist for real-time tracking. Consider a

761 problematic large carnivore (e.g., lion or bear) residing in a national park. Rather than

762 relocating or exterminating this animal, a real-time tracking system could be used to 
763 monitor the animal's movements. Park managers could use this information to improve

764 park safety and minimize human-animal conflicts through trail/site closures and

765 surveillance efforts.

766 Further developments with real-time movement data will involve the creation of 767 increasingly sophisticated models for predicting future movement locations. The space-

768 time cone from time geography (see Figure 1a) provides only the boundary for future

769 movement possibilities (e.g., O’Sullivan et al. 2000), factoring in the uneven distribution

770 of future movement possibilities (e.g., Winter 2009) provides more useful information for

771 prediction. Future movement possibilities can be linked to contextual factors such as

772 obstacles (Prager 2007), underlying movement cost surfaces (Miller and Bridwell 2009),

773 and object kinetics (Kuijpers et al. 2011). Further developments towards probabilistically

774 predicting future movements based on contextual factors will provide researchers and

775 analysts with powerful tools for linking real-time movement data with other data sources.

776 With human movement data a new field that is gaining momentum focuses on

777 leveraging real-time location data in everyday applications: location based services

778 (Raper et al. 2007). Location based services have developed coincidentally with the

779 availability of location-aware devices (e.g., GPS enabled cell-phones and handheld

780 devices), which are now integral to people's daily routines (Kumar and Stokkeland

781 2003). However, given the revealing nature of personal movement data, concerns over

782 the privacy and ownership rights of personal movement information continue to surface

783 (e.g., Dobson and Fischer 2003). With location based services, the fundamental goal is to

784 tailor individual applications, services, and marketing to a user's real-time location

785 (Raper et al. 2007). For example, methods for predicting future movements based on 
786 contextual factors, when applied in a real-time application, could provide increased

787 functionality and improve user experiences with location based services. As methods for

788 analyzing real-time movement data emerge, their development in conjunction with

789 applications from location based services should be conducted in order to facilitate their

790 adoption in this field.

791 With the development of technologies for acquiring movement data, the ability to 792 capture finely grained movement data has increased substantially. Opportunities exist for

793 investigating properties of movement previously not feasible with coarser grained

794 movement data. For example, investigating velocities, accelerations, and the role of

795 momentum in moving objects is an area of opportunity. Current research is developing

796 methods for incorporating physical kinetics (based on object velocity and acceleration)

797 into the calculation of time geography volumes, such as those from Figure 1 (Kuijpers et

798 al. 2011). Another avenue for future work is the development of a probabilistic time

799 geographic framework, such as by Winter (2009), that considers the influence of kinetics

800 into the calculation of future movement probabilities.

$801 \quad$ Methods for investigating interactions between individuals in groups of moving

802 objects continue to develop, but remain limited in overall scope and sophistication. Laube

803 et al. (2005)'s relative motion concept can identify trendsetters, but uses only movement

804 azimuth in its derivation. Others have developed other ways to identify specific types of

805 interactions between moving individuals (e.g., Andersson et al. 2008; Buchin et al. 2010).

806 As our ability to characterize these patterns grows, it may be more useful to investigate

807 methods for quantifying the strength of interactions that occur in movement databases.

808 That is, can we measure how interactive are the movements of two individuals. The work 
809 of Shirabe (2006) provides a necessary starting point for this research which could be

810 further investigated in light of this problem. Further, it may be necessary to examine

811 outside factors influencing the levels of interaction between individuals (e.g., barriers and

812 obstacles represented as lines/polygons, Noyon et al. 2007). Subsequently, how to

813 accommodate other data sources into models for measuring individual level interactions

814 in movement data remains an open research problem.

815 With time geography, Hägerstrand provided a theoretical context for looking at

816 the constraints of object movement. Contemporary geographers continue to expand on

817 time geographic concepts incorporating a range of ideas into time geographic theory

818 (e.g., Winter 2009, Miller and Bridwell 2009, Delafontaine et al. 2011b). As discussed by

819 Lenntorp (1999), Hägerstrand's time geography represents a set of conceptual and

820 methodological building blocks for use in analyzing and understanding movement as a

821 process. As the quantitative toolkit for analyzing movement continues to grow and

822 develop, those methods including theory and ideas from time geography in their

823 derivation will have increased value in a broader range of applications.

824 Other theoretical frameworks have also been successfully implemented in

825 movement research. For example, the idea that movement is motivated by an underlying

826 field (e.g., Brillinger et al. 2001) suggests that forces of attraction and repulsion may

827 influence movements. Such points of attraction, for example in wildlife, may be used to

828 investigate central place foraging theory (Orians and Pearson 1979). Markovian models

829 have also been used to demonstrate how movement operates as a diffusion process (e.g.,

830 Skellum 1951). Diffusion, originally used to describe random dispersal of organisms, can

831 also be related to crowd dynamics in humans (Batty et al. 2003). The use of theoretical 
832 constructs in quantitative methods, such as the aforementioned examples, demonstrates

833 thoughtful development of ideas that in the end are easier to interpret for both the reader

834 and analyst.

835 It has been suggested that movement methods must consider the "geography

836 behind trajectories" (Bogorny et al. 2009) in order to understand the geographic

837 processes affecting observed movement patterns. Movement analysis is no longer limited

838 by available data, but rather by the tools required to manage and analyze movement

839 databases in more efficient and sophisticated ways (Miller 2010). Thus, the continued

840 development of methods capable of integrating increasingly large and complex

841 movement databases with available spatial and temporal layers is warranted. With such

842 analysis, the goal is to identify relationships between movement patterns and underlying

843 spatial and/or temporal variables. Data mining work is beginning to enrich movement

844 data with underlying geographic datasets (Alvares et al. 2007, Bogorny et al. 2009).

845 Quantitative methods for movement data must be further developed to consider

846 underlying geographic variables in order for movement to be understood as a function of

847 the environment. Similarly, novel movement datasets are emerging where attribute data

848 are recorded along with spatial and temporal records (e.g., $\langle I D, S, T, A>$, where $A$

849 represents some attribute data). For example, wildlife tracking systems are being

850 equipped with devices, such as cameras (Hunter et al. 2005), that simultaneously record

851 information alongside movement fixes. The inclusion of attributes with movement fixes

852 can be termed marked movement data, comparable to the term marked point pattern in

853 the spatial statistics literature (Cressie 1993). Inclusion of attributes (numerical or

854 categorical) alongside spatial locations in movement data represents an area of 
855 opportunity for advanced analysis in the movement-attribute space, as existing methods

856 are not designed for marked movement data.

\section{$858 \quad 6-$ Conclusions}

859 Novel movement datasets are not only becoming readily available they are

860 changing how data on movement processes are captured. Traditionally, movement data

861 have been collected as samples taken at coarse temporal granularities. Coarsely collected

862 movement data represents movement discretely and with considerable uncertainty

863 between sampled points. More recently, movement data are being collected at extremely

864 fine temporal granularities, such as 5 fixes/second with athletes. Finely grained

865 movement data represents a (near) continuous form of movement data which contains

866 minimal uncertainty in space-time location. Not only are existing methods ill-suited for

867 finely grained movement data, but the types of questions being asked must also be

868 revisited to consider that uncertainty between consecutive fixes is negligible.

869 Within GIS data formats, there is a clear lack of appropriate structures for

870 handling movement data. Those interested in purely visualizing movement data have

871 circumvented these problems by generating independent platforms for visualizations

872 (Andrienko et al. 2005). However, the development of quantitative methods is still

873 hindered by difficulties representing the temporal domain within GIS. The development

874 of geospatial data formats exclusively for movement data will invigorate future research

875 into quantitative methods for movement.

876 There is a clear need for novel quantitative methods for extracting information

877 and generating knowledge from ever-expanding movement datasets (Wolfer et al. 2001, 
878 Laube et al. 2007). Most existing methods can be classified as data mining algorithms,

879 which are used to identify and categorize trends in movement databases, based on some $a$ 880 priori notion about movement. Emerging problems investigate more complex patterns

881 and relationships contained in movement datasets, such as the identification of flocking

882 behavior (Benkert et al. 2008). Methods that are able to quantify interactions between

883 individuals (Laube et al. 2005), and with environmental variables (Patterson et al. 2009)

884 in movement databases will be increasingly relevant in more sophisticated movement

885 analyses. Movement models capable of quantifying relationships between moving objects

886 and dynamic features in the environment (e.g., traffic conditions) are justified in order to

887 measure the significance of events or changes on object movement.

889 Acknowledgements

890 The authors would like to thank the referees for their constructive remarks on earlier

891 versions of this manuscript. Their comments greatly improved the presentation of this

892 work. Support for this work was obtained from the Natural Sciences and Engineering

893 Research Council of Canada, and GEOIDE through the Government of Canada's

894 Networks for Centres of Excellence program. 
897

898

899

900

901

902

903

904

905

906

907

908

909

910

911

912

913

914

915

916

917

918

919

920

921

922

923

924

925

926

927

928

929

930

931

932

933

934

935

936

937

938

939
Ahas, R., et al. 2007. Seasonal tourism spaces in Estonia: Case study with mobile positioning data. Tourism Management, 28, 898-910.

Alt, H. and Godau, M. 1995. Computing the Fréchet distance between two polygonal curves. International Journal of Computational Geometry \& Applications, 5(1-2), 75-91.

Alvares, L.O., et al., 2007. A model for enriching trajectories with semantic geographical information. GIS'07: Proceedings of the 15th Annual ACM International Symposium on Advances in Geographic Information Systems, New York, NY, 8.

Andersson, M., et al. 2008. Reporting leaders and followers among trajectories of moving point objects. Geoinformatica, 12, 497-528.

Andrews, H.F. 1973. Home range and urban knowledge of school-age children. Environment and Behaviour, 5, 73-86.

Andrienko, N. and Andrienko, G. 2007. Designing visual analytics methods for massive collections of movement data. Cartographica, 42(2), 117-138.

Andrienko, N., Andrienko, G. and Gatalsky, P., 2005. Impact of data and task characteristics on design of spatio-temporal data visualization tools. In: Dykes, J.A., MacEachren, A.M. and Kraak, M.J. eds. Exploring Geovisualization. New York: Elsevier, 201-222.

Andrienko, G. et al., 2010. GeoVA(t) - Geospatial visual analytics: Focus on time. International Journal of Geographical Information Science, 24(10), 1453-1457.

Anselin, L. 1995. Local indicators of spatial association - LISA. Geographical Analysis, 27(2), 93-115.

Atev, S., Masoud, O. and Papanikolopoulos, N., 2006. Learning traffic patterns at intersections by spectral clustering of motion trajectories. IEEE/RSJ International Conference on Intelligent Robots and Systems, Oct. 9-15, Beijing, China, 48514856.

Baer, L.D. and Butler, D.R. 2000. Space-time modeling of grizzly bears. Geographical Review, 90(2), 206-221.

Batty, M., Desyllas, J. and Duxbury, E. 2003. The discrete dynamics of small-scale events: agent-based models of mobility in carnivals and street parades. International Journal of Geographical Information Science, 17(7), 673-697.

Benhamou, S. 2004. How to reliably estimate the tortuosity of an animal's path: straightness, sinuosity, or fractal dimension? Journal of Theoretical Biology, 229, 209-220.

Benkert, M., et al., 2007. Finding popular places. In: Tokuyama, T., ed. ISAAC 2007, LNCS 4835, 776-787.

Benkert, M., et al. 2008. Reporting flock patterns. Computational Geometry, 41, 111125.

Bogorny, V., Kuijpers, B. and Alvares, L.O. 2009. ST-DMQL: A semantic trajectory data mining query language. International Journal of Geographical Information Science, 23(10), 1245-1276.

Boots, B. 2002. Local measures of spatial association. Ecoscience, 9(2), 168-176. 
940

941

942

943

944

945

946

947

948

949

950

951

952

953

954

955

956

957

958

959

960

961

962

963

964

965

966

967

968

969

970

971

972

973

974

975

976

977

978

979

980

981

982

983
Borruso, G. 2008. Network density estimation: a GIS approach for analysing point patterns in a network space. Transactions in GIS, 12(3), 377-402.

Brillinger, D.R. 2007. A potential function approach to the flow of play in soccer. Journal of Quantitative Analysis in Sports, 3(1), Article 3.

Brillinger, D.R., et al., 2001. The use of potential functions in modelling animal movement. In: Saleh, M.A.K. ed. Data Analysis from Statistical Foundations. Huntington, NY: Nova Science Publishers Ltd., 369-386.

Brillinger, D.R., et al. 2002. Employing stochastic differential equations to model wildlife motion. Bulletin Brazilian Mathematical Society, 33(3), 385-408.

Brillinger, D.R., et al. 2004. An exploratory data analysis (EDA) of the paths of moving animals. Journal of Statistical Planning and Inference, 122, 43-63.

Buchin, K., Buchin, M. and Gudmundsson, J. 2010. Constrained free space diagrams: a tool for trajectory analysis. International Journal of Geographical Information Science, 24(7), 1101-1125.

Burns, L.D., 1979. Transportation, Temporal, and Spatial Components of Accessibility. Lexington, MA: Lexington Books.

Burt, W.H. 1943. Territoriality and home range concepts as applied to mammals. Journal of Mammalogy, 24(3), 346-352.

Calenge, C., Dray, S. and Royer-Carenzi, M. 2009. The concept of animals' trajectories from a data analysis perspective. Ecological Informatics, 4, 34-41.

Casaer, J., et al. 1999. Analysing space use patterns by Thiessen polygon and triangulated irregular network interpolation: a non-parametric method for processing telemetric animal fixes. International Journal of Geographical Information Science, 13(5), 499-511.

Chrisman, N.R., 1998. Beyond the snapshot: Changing the approach to change, error, and process. In: Egenhofer, M.J. and Golledge, R. eds. Spatial and Temporal Reasoning in Geographic Information Systems. New York, NY: Oxford University Press, 85-93.

Claussen, D.L., Finkler, M.S. and Smith, M.M. 1997. Thread trailing of turtles: methods for evaluating spatial movements and pathway structure. Canadian Journal of Zoology, 75, 2120-2128.

Cliff, A.D. and Ord, J.K., 1973. Spatial Autocorrelation. London: Pion Limited.

Cliff, A.D. and Ord, J.K., 1981. Spatial Processes: Models and Applications. London: Pion.

Cressie, N., 1993. Statistics for Spatial Data. New York: John Wiley \& Sons Inc.

D'Auria, M., Nanni, M. and Pedreschi, D., 2005. Time-focused density-based clustering of trajectories of moving objects. Proceeding of the Workshop on Mining Spatiotemporal Data (MSTD-2005), Porto, 14.

Delafontaine, M., et al., 2011a. The impact of opening hours on the equity of individual space-time accessibility. Computers, Environment and Urban Systems, 35(4), pp.276-288.

Delafontaine, M., Neutens, T. and Van De Weghe, N., 2011b. Modelling potential movement in constrained travel environments using rough space - time prisms. International Journal of Geographical Information Science, 25(9), 1389-1411. 
984

985

986

987

988

989

990

991

992

993

994

995

996

997

998

999

1000

1001

1002

1003

1004

1005

1006

1007

1008

1009

1010

1011

1012

1013

1014

1015

1016

1017

1018

1019

1020

1021

1022

1023

1024

1025

1026

1027

1028

1029
Demšar, U. and Virrantaus, K. 2010. Space -time density of trajectories: exploring spatiotemporal patterns in movement data. International Journal of Geographical Information Science, 24(10), 1527-1542.

Dettki, H., Ericsson, G. and Edenius, L. 2004. Real-time moose tracking: An internet based mapping application using GPS/GSM-collars in Sweden. Alces, 40, 13-21.

Dicke, M. and Burrough, P.A. 1988. Using fractal dimensions for characterizing tortuosity of animal trails. Physiological Entomology, 13, 393-398.

Diggle, P.J., 2003. Statistical Analysis of Spatial Point Patterns. 2nd ed. New York, NY: Arnold.

Dixon, K.R. and Chapman, J.A. 1980. Harmonic mean measure of animal activity areas. Ecology, 61(5), 1040-1044.

Dobson, J.E., and Fisher, P., 2003. Geoslavery. IEEE Technology and Society Magazine, 22(1), 47-52.

Dodge, S., Laube, P. and Weibel, R., 2012. Movement similarity assessment using symbolic representation of trajectories. International Journal of Geographical Information Science. DOI:10.1080/13658816.2011.630003, 26p.

Dodge, S., Weibel, R. and Lautenschutz, A.-K. 2008. Towards a taxonomy of movement patterns. Information Visualization, 7, 240-252.

Douglas, D.H. 1994. Least-cost path in GIS using an accumulated cost surface and slopelines. Cartographica, 31(3), 37-51.

Downs, J.A., 2010. Time-geographic density estimation for moving point objects. In: Fabrikant, S.I., et al., eds. GIScience 2010, LNCS 6292, Sep. 14-17, Zurich, Switzerland, 16-26.

Downs, J.A. and Horner, M.A. 2009. A characteristic-hull based method for home range estimation. Transactions in GIS, 13(5-6), 527-537.

Downs, J.A., Horner, M.W. and Tucker, A.D., 2012. Time-geographic density estimation for home range analysis. Annals of GIS, 17(3), 163-171.

Drewe, P., 2005. What about time in urban planning \& design in the ICT age? CORP 2005 \& Geomultimedia05, Feb. 22-25, Vienna, Austria, 13-37.

Duda, R.O., Hart, P.E. and Stork, D.G., 2001. Pattern Classification. New York: John Wiley \& Sons.

Dungan, J.L., et al. 2002. A balanced view of scale in spatial statistical analysis. Ecography, 25(5), 626-640.

Dykes, J., MacEachren, A.M. \& Kraak, M.J. eds., 2005. Exploring Geovisualization. 1st ed., San Diego, CA: Elsevier.

Dykes, J.A. and Mountain, D.M. 2003. Seeking structure in records of spatio-temporal behaviour: visualization issues, efforts and applications. Computational Statistics \& Data Analysis, 43, 581-603.

Eagle, N. and Pentland, A.S. 2009. Eigenbehaviors: identifying structure in routine. Behavioral Ecology and Sociobiology, 63, 1057-1066.

Eiter, T. and Mannila, H., 1994. Computing discrete Fréchet distance. Technical University of Wien, CD-TR 94/64.

Erwig, M., et al. 1999. Spatio-temporal data types: An approach to modeling and querying moving objects in databases. Geoinformatica, 3(3), 269-296.

Forer, P., 1998. Geometric approaches to the nexus of time, space, and microprocess: Implementing a practical model for mundane socio-spatial systems. In: 
Egenhofer, M.J. and Golledge, R.G. eds. Spatial and Temporal Reasoning in Geographic Information Systems. New York: Oxford University Press, 171-190.

Frank, A.U., 1998. Different types of "times" in GIS. In: Egenhofer, M.J. and Golledge, R.G. eds. Spatial and Temporal Reasoning in Geographic Information Systems. New York: Oxford University Press, 40-62.

Gao, Y., et al. 2010. Algorithms for constrained $k$-nearest neighbor queries over moving object trajectories. Geoinformatica, 14, 241-276.

Giannotti, F., et al., 2007. Trajectory pattern mining. Knowledge Discovery and Data Mining, August 12-15, San Jose, CA, USA, 330-339.

Goodchild, M.F. 1992. Geographical information science. International Journal of Geographical Information Systems, 6(1), 31-45.

Gudmundsson, J. and van Kreveld, M., 2006. Computing longest duration flocks in trajectory data. ACM-GIS'06, Nov. 10-11, Arlington, VA, USA, 8.

Gudmundsson, J., Van Kreveld, M. and Speckmann, B. 2007. Efficient detection of patterns in 2D trajectories of moving points. Geoinformatica, 11, 195-215.

Güting, R.H., Behr, T. and Christian, D., 2010a. SECONDO: A platform for moving objects database research and for publishing and integrating research implementations. Bulletin of the IEEE Computer Society Technical Committee on Data Engineering, 33(2), 56-63.

Güting, R.H., Behr, T. and Xu, J. 2010b. Efficient $k$-nearest neighbor search on moving object trajectories. The VLDB Journal, 19, 687-714.

Güting, R.H., et al. 2000. A foundation for representing and querying moving objects. ACM Transactions on Database Systems, 25(1), 1-42.

Güting, R.H. and Mamoulis, N., 2011. Special issue on data management for mobile services. The VLDB Journal, 20(5), 641-642.

Güting, R.H. and Schneider, M., 2005. Moving Objects Databases. New York, NY: Elsevier.

Hadjieleftheriou, M., et al. 2003. On-line discovery of dense areas in spatio-temporal databases. LNCS 2750, 306-325.

Hägerstrand, T. 1970. What about people in regional science? Papers of the Regional Science Association, 24, 7-21.

Hallin, P.O. 1991. New paths for time-geography? Geografiska Annaler, 73(3), 199-207.

Hemson, G., et al. 2005. Are kernels the mustard? Data from global positioning system (GPS) collars suggests problems for kernel home-range analyses with leastsquares cross-validation. Journal of Animal Ecology, 74, 455-463.

Hengl, T., et al., 2008. Geostatistical analysis of GPS trajectory data: space-time densities. Proceedings of the 8th International Symposium on Spatial Accuracy Assessment in Natural Resources and Environmental Sciences, Shanghai, June 25-27, 17-24.

Holly, B.P., 1978. The problem of scale in time-space research. In: Carlstein, T., Parkes, D.N. and Thrift, N. eds. Time and Regional Dynamics. New York: John Wiley \& sons, 5-18.

Horne, J.S., et al. 2007. Analyzing animal movement using Brownian bridges. Ecology, 88(9), 2354-2363.

Hornsby, K. and Egenhofer, M.J. 2002. Modeling moving objects over multiple granularities. Annals of Mathematics and Artificial Intelligence, 36, 177-194. 
1076

1077

1078

1079

1080

1081

1082

1083

1084

1085

1086

1087

1088

1089

1090

1091

1092

1093

1094

1095

1096

1097

1098

1099

1100

1101

1102

1103

1104

1105

1106

1107

1108

1109

1110

1111

1112

1113

1114

1115

1116

1117

1118

1119

1120

Hunter, A., El-Sheimy, N. and Stenhouse, G.B. 2005. Up close and grizzly: GPS/camera collar captures bear doings. GPS World, Feb. 2005, 24-31.

Huttenlocher, D.P., Klanderman, G.A. and Rucklidge, W.J. 1993. Comparing images using the Hausdorff distance. IEEE Transactions on Pattern Analysis and Machine Intelligence, 15(9), 850-863.

Hwang, J.-R., Kang, H.-Y. and Li, K.-J., 2005. Spatio-temporal similarity analysis between trajectories on road networks. In: Akoka, J., et al., eds. Perspectives in Conceptual Modeling, ER Workshops, LNCS 3770, 280-289.

Jennrich, R.I. and Turner, F.B. 1969. Measurement of non-circular home range. Journal of Theoretical Biology, 22, 227-237.

Jensen, C.S., Lu, H. and Yang, B., 2010. Indoor - A new data management frontier. Bulletin of the IEEE Computer Society Technical Committee on Data Engineering, 33(2), 12-17.

Jeung, H., et al., 2008. Discovery of convoys in trajectory databases. Proceedings VLDB, Auckland, New Zealand, Aug. 23-28, 1068-1080.

Johnston, R.J. 1997. W(h)ither spatial science and spatial analysis. Futures, 29(4/5), 323336.

Jonsen, I.D., Myers, R.A. and Mills Flemming, J. 2003. Meta-analysis of animal movement using state-space models. Ecology, 84(11), 3055-3063.

Kalnis, P., Mamoulis, N. and Bakiras, S., 2005. On discovering moving clusters in spatiotemporal data. In: Medeiros, C.B., Egenhofer, M.J. and Bertino, E., eds. 9th International Symposium on Advances in Spatial and Temporal Databases, LNCS 3633, 364-381.

Kareiva, P.M. and Shigesada, N. 1983. Analyzing insect movement as a correlated random walk. Oecologia, 56, 234-238.

Kie, J.G., et al. 2010. The home-range concept: are traditional estimators still relevant with modern telemetry technology? Philosophical Transactions of the Royal Society B, 365, 2221-2231.

Kondo, K. and Kitamura, R. 1987. Time-space constraints and the formation of trip chains. Regional Science and Urban Economics, 17, 49-65.

Kraak, M.J., 2003. The space-time cube revisited from a geovisualization perspective. Proceedings 21 st International Cartographic Conference, August 10-16, Durban, South Africa, 1988-1995.

Kuijpers, B., Miller, H.J. and Othman, W., 2011. Kinetic space-time prisms. In 19th ACM SIGSPATIAL International Conference on Advances in Geographic Information Systems. New York, NY: Association of Computing Machinery, 162-170.

Kumar, S. and Stokkeland, J. 2003. Evolution of GPS technology and its subsequent use in commercial markets. International Journal of Mobile Communications, 1(1/2), 180-193.

Kwan, M.P. 1998. Space-time and integral measures of individual accessibility: A comparative analysis using a point-based framework. Geographical Analysis, 30(3), 191-216.

Kwan, M.P. 2004. GIS methods in time-geographic research: Geocomputation and geovisulization of human activity patterns. Geografiska Annaler, 86 B(4), 267280. 
1121

1122

1123

1124

1125

1126

1127

1128

1129

1130

1131

1132

1133

1134

1135

1136

1137

1138

1139

1140

1141

1142

1143

1144

1145

1146

1147

1148

1149

1150

1151

1152

1153

1154

1155

1156

1157

1158

1159

1160

1161

1162

1163

1164

1165

1166
Langran, G. and Chrisman, N.R. 1988. A framework for temporal geographic information. Cartographica, 25(3), 1-14.

Laube, P., et al. 2007. Movement beyond the snapshot - Dynamic analysis of geospatial lifelines. Computers, Environment and Urban Systems, 31, 481-501.

Laube, P., Imfeld, S. and Weibel, R. 2005. Discovering relative motion patterns in groups of moving point objects. International Journal of Geographical Information Science, 19(6), 639-668.

Laube, P. and Purves, R.S. 2011. How fast is a cow? Cross-scale analysis of movement data. Transactions in GIS, 15(3), 401-418.

Laube, P., van Kreveld, M. and Imfeld, S., 2004. Finding REMO - Detecting relative motion patterns in geospatial lifelines. In: Fisher, P.F. ed. Developments in Spatial Data Handling, Proceedings of the 11th International Symposium on Spatial Data Handling. Berlin: Springer, 201-215.

Laver, P.N. and Kelly, M.J. 2008. A critical review of home range studies. Journal of Wildlife Management, 72(1), 290-298.

Lenntorp, B., 1976. Paths in Space-Time Environments: a Time Geographic Study of Movement Possibilities of Individuals. Stockholm: Gleerup.

Lenntorp, B. 1999. Time-geography - at the end of its beginning. GeoJournal, 48, 155158.

Long, J.A. and Nelson, T.A. 2012. Time geography and wildlife home range delineation. Journal of Wildlife Management,76(2), 407-413.

Mark, D.M., 1998. Geospatial lifelines. Integrating Spatial and Temporal Databases, 98471, Dagstuhl Seminars, 12.

Mennis, J., Viger, R. and Tomlin, C.D. 2005. Cubic map algebra functions for spatiotemporal analysis. Cartography and Geographic Information Science, 32(1), 1732.

Miller, H.J. 1991. Modelling accessibility using space-time prism concepts within geographical information systems. International Journal of Geographical Information Systems, 5(3), 287-301.

Miller, H.J. 2003. What about people in geographic information science? Computers, Environment and Urban Systems, 17, 447-453.

Miller, H.J. 2005. A measurement theory for time geography. Geographical Analysis, 37, 17-45.

Miller, H.J. 2010. The data avalanche is here. Shouldn't we be digging? Journal of Regional Science, 50(1), 181-201.

Miller, H.J. and Bridwell, S.A. 2009. A field-based theory for time geography. Annals of the Association of American Geographers, 99(1), 49-75.

Miller, H.J. and Wu, Y.H. 2000. GIS Software for measuring space-time accessibility in transportation planning and analysis. Geoinformatica, 4(2), 141-159.

Nanni, M. and Pedreschi, D. 2006. Time-focused clustering of trajectories of moving objects. Journal of Intelligent Information Systems, 27, 267-289.

Nathan, R., et al. 2008. A movement ecology paradigm for unifying organismal movement research. Proceedings of the National Academy of Science, 105(49), 19052-19059.

Neutens, T., et al. 2007. Human interaction spaces under uncertainty. Transportation Research Record, 2021, 28-35. 
Neutens, T. et al., 2010. Evaluating the temporal organization of public service provision using space-time accessibility analysis. Urban Geography, 31(8), 1039-1064.

Noyon, V., Claramunt, C. and Devogele, T. 2007. A relative representation of trajectories in geographical spaces. Geoinformatica, 11, 479-496.

O'Sullivan, D., Morrison, A. and Shearer, J. 2000. Using desktop GIS for the investigation of accessibility by public transport: an isochrone approach. International Journal of Geographical Information Science, 14(1), 85-104. O'Sullivan, D. and Unwin, D.J., 2010. Geographic Information Analysis. 2nd ed. Hoboken, NJ: John Wiley \& Sons.

Okabe, A., Satoh, T. and Sugihara, K. 2009. A kernel density estimation method for networks, its computational method and a GIS-based tool. International Journal of Geographical Information Science, 23(1), 7-32.

Openshaw, S., 1984. The Modifiable Areal Unit Problem. Norwich, England: GeoBooks.

Orians, G.H. and Pearson, N.E., 1979. On the theory of central place foraging. In: Horn, D.J., Stairs, G.R. and Mitchell, R.D. eds. Analysis of Ecological Systems. Columbus, OH: Ohio State University Press, 155-178.

Palma, A.T., et al., 2008. A clustering-based approach for discovering interesting places in trajectories. Proceedings of the 2008 ACM Symposium on Applied Computing, Fortaleza, Ceara, Brazil, 6.

Parkes, D.N. and Thrift, N. 1975. Timing space and spacing time. Environment and Planning A, 7, 651-670.

Patterson, T.A., et al. 2009. Classifying movement behaviour in relation to environmental conditions using hidden Markov models. Journal of Animal Ecology, 78, 11131123.

Peuquet, D.J. 1994. It's about time: a conceptual framework for the representation of temporal dynamics in geographic information systems. Annals of the Association of American Geographers, 84(3), 441-461.

Peuquet, D.J., 2002. Representations of Space and Time. New York, NY: The Guilford Press.

Pfoser, D., Jensen, C.S. and Theodoridis, Y., 2000. Novel approaches to the indexing of moving object trajectories. 26th Conference on Very Large Databases, Cairo, Egypt, Sep. 10-14, 12p.

Prager, S.D., 2007. Environmental contextualization of uncertainty for moving objects. Computers, Environment and Urban Systems, 31(3), 303-316.

Pred, A., ed., 1981. Space and Time in Geography. Gleerup: The Royal University of Lund.

Pultar, E., et al. 2010. EDGIS: a dynamic GIS based on space time points. International Journal of Geographical Information Science, 24(3), 329-346.

Raper, J., 2002. The dimensions of GIScience. Second International Conference of Geographic Information Science, GIScience 2002., Boulder, CO, Last retrieved: May 2011, <http://www.soi.city.ac.uk/ raper/research/GIScience2002-OHspub.ppt>.

Raper, J., et al. 2007. A critical evaluation of location based services and their potential. Journal of Location Based Services, 1(1), 5-45.

Righton, D. and Mills, C. 2006. Application of GIS to investigate the use of space in coral reef fish: a comparison of territorial behaviour in two Red Sea 
butterflyfishes. International Journal of Geographical Information Science, 20(2), 215-232.

Rose, C. 1977. Reflections on the notion of time incorporated in Hägerstrand's timegeographic model of society. Tijdschrift voor Economische en Sociale Geografie, 68(1), 43-50.

Sanderson, G.C. 1966. The study of mammal movements: A review. Journal of Wildlife Management, 30(1), 215-235.

Shao, F., Cai, S. and Gu, J., 2010. A modified Hausdorff distance based algorithm for 2dimensional spatial trajectory matching. The 5th International Conference on Computer Science \& Education, Aug. 24-27, Hefei, China, 166-172.

Shaw, S.L., Yu, H. and Bombom, L.S. 2008. A space-time GIS approach to exploring large individual-based spatiotemporal datasets. Transactions in GIS, 12(4), 425441.

Sheppard, E. 2001. Quantitative geography: representations, practices, and possibilities. Environment and Planning D: Society and Space, 19, 535-554.

Shirabe, T., 2006. Correlation analysis of discrete motions. In: Raubal, M., et al. eds. GIScience 2006. LNCS, vol. 4197. Berlin: Springer-Verlag, 370-382.

Shoshany, M., Even-Paz, A. and Bekhor, S. 2007. Evolution of clusters in dynamic point patterns: with a case study of Ants' simulation. International Journal of Geographical Information Science, 21(7), 777-797.

Shoval, N. and Isaacson, M. 2007. Sequence alignment as a method for human activity analysis in space and time. Annals of the Association of American Geographers, 97(2), 282-297.

Silverman, B.W., 1986. Density Estimation for Statistics and Data Analysis. New York: Chapman and Hall.

Sinha, G. and Mark, D.M. 2005. Measuring similarity between geospatial lifelines in studies of environmental health. Journal of Geographical Systems, 7, 115-136.

Skellum, J.G. 1951. Random dispersal in theoretical populations. Biometrika, 38, 196218.

Spaccapietra, S., et al. 2008. A conceptual view on trajectories. Data \& Knowledge Engineering, 65, 126-146.

Stewart Hornsby, K. and Cole, S. 2007. Modeling moving geospatial objects from an event-based perspective. Transactions in GIS, 11(4), 555-573.

Thomas, J.J. and Cook, K.A., 2005. Illuminating the Path: The Research and Development Agenda for Visual Analytics. National Visualization and Analytics Center. $184 \mathrm{p}$.

Tremblay, Y., et al. 2006. Interpolation of animal tracking data in a fluid environment. The Journal of Experimental Biology, 209, 128-140.

Turchin, P., 1998. Quantitative Analysis of Movement: Measuring and Modelling Population Redistribution in Animals and Plants. Sunderland, MA: Sinauer.

Verhein, F. and Chawla, S., 2006. Mining spatio-temporal association rules, sources, sinks, stationary regions and thoroughfares in object mobility databases. Proceedings of the 11th International Conference on Database Systems for Advanced Applications (DASFAA), 187-201.

Verhein, F. and Chawla, S. 2008. Mining spatio-temporal patterns in object mobility databases. Data Mining \& Knowledge Discovery, 16, 5-38. 
1259

1260

1261

1262

1263

1264

1265

1266

1267

1268

1269

1270

1271

1272

1273

1274

1275

1276

1277

1278

1279

1280

1281

1282

1283

1284

1285

1286

1287

1288
Viswanathan, G.M., et al. 1996. Lévy flight search patterns of wandering albatrosses. Nature, 381, 413-415.

Vlachos, M., Gunopulos, D. and Kollios, G., 2002. Robust similarity measures for mobile object trajectories. 5th International Workshop on Mobility in Databases and Distributed Systems (MDDS), Aix-en-Provence, France, 721-726.

Wells, L.L., 1981. Real-time missile tracking with GPS. National Aerospace Meeting, Apr. 8-10, Washington, DC, 56-61.

Wiens, J.A., Crist, T.O. and Milne, B.T. 1993. On quantifying insect movements. Environmental Entomology, 22(4), 709-715.

Winter, S., 2009. Towards a probabilistic time geography. In: Mokbel, M., Scheuermann, P. and Aref, W.G., eds. Proceedings of the 17th ACM SIGSPATIAL International Conference on Advances in Geographic Information Systems, Seattle, WA, 528531.

Winter, S. and Yin, Z.C. 2010. Directed movements in probabilistic time geography. International Journal of Geographical Information Science, 24(9), 1349-1365.

Wolfer, D.P., et al. 2001. Extended analysis of path data from mutant mice using the public domain software Wintrack. Physiology \& Behavior, 73, 745-753.

Worton, B.J. 1989. Kernel methods for estimating the utilization distribution in homerange studies. Ecology, 70(1), 164-168.

Yanagisawa, Y., Akahani, J.I. and Satoh, T., 2003. Shape-based similarity query for trajectory of mobile objects. In: Chen, M.-S., et al. eds. Mobile Data Management, LNCS 2574. Berlin: Springer-Verlag, 63-77.

$\mathrm{Yu}$, B. and Kim, S.H., 2006. Interpolating and using most likely trajectories in movingobjects databases. In: Bressan, S., Kung, J. and Wagner, R., eds. DEXA 2006, LNCS 4080, 718-727.

Zhang, Z., Huang, K. and Tan, T., 2006. Comparison of similarity measures for trajectory clustering in outdoor surveillance scenes. IEEE International Conference on Pattern Recognition, 1135-1138. 
Table 1: Terms used synonymously for describing movement data.

\begin{tabular}{lll}
\hline Description & Term & Synonymous terms (with selected references) \\
\hline $\begin{array}{l}\text { A single record of object movement (of } \\
\text { the form }<\mathrm{ID}, \mathrm{S}, \mathrm{T}>\text { ). }\end{array}$ & $\begin{array}{l}\text { Movement Fix } \\
\left(M_{t}\right)\end{array}$ & $\begin{array}{l}\text { point, observation, relocation } \\
\end{array}$ \\
\hline $\begin{array}{l}\text { A sequence of ordered records in time } \\
\text { depicting the movement of a single } \\
\text { object. }\end{array}$ & $\begin{array}{l}\text { Movement } \\
\text { Path }\left(M^{a}\right)\end{array}$ & $\begin{array}{l}\text { space-time path (Hägerstrand 1970), trip-chain } \\
\text { (Kondo and Kitamura 1987), geospatial lifeline } \\
\text { (Mark 1998), trajectory, trace, track }\end{array}$ \\
\hline $\begin{array}{l}\text { A collection of records depicting the } \\
\text { movements of many objects or the same } \\
\text { object at different occasions, potentially } \\
\text { including attribute information. }\end{array}$ & Movement & $\begin{array}{l}\text { moving objects database (Güting and Schneider } \\
\text { 2005) }\end{array}$ \\
\hline
\end{tabular}


Table 2: Parameters extractable from movement data sorted by dimension. After Table 1 from Dodge et al. (2008).

\begin{tabular}{cccc}
\hline & Primitive & Primary Derivatives & Secondary Derivatives \\
\hline $\begin{array}{c}\text { Spatial } \\
(\mathbf{x}, \mathbf{y})\end{array}$ & Position & Distance & Spatial distribution \\
& & Direction & Change of direction \\
Temporal & Spatial extent & Sinuosity \\
$(\mathbf{t})$ & Instance & Duration & Temporal distribution \\
\hline $\begin{array}{c}\text { Spatio- } \\
\text { temporal } \\
(\mathbf{x}, \mathbf{y}, \mathbf{t})\end{array}$ & - & Travel time & Change of duration \\
\hline
\end{tabular}




\section{Figure Captions}

Figure 1: Volumes used in Hägerstrand's time geography: a) space-time cone, b) spacetime prism, c) potential path area, and d) path bundling.

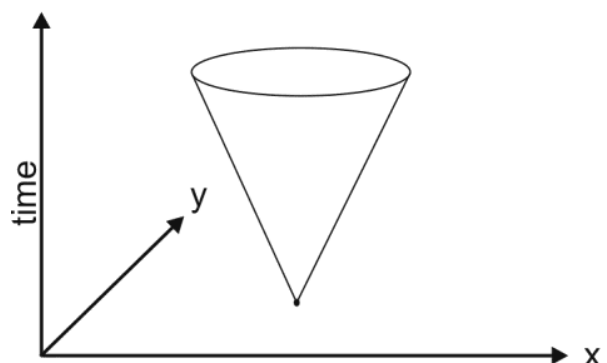

a)

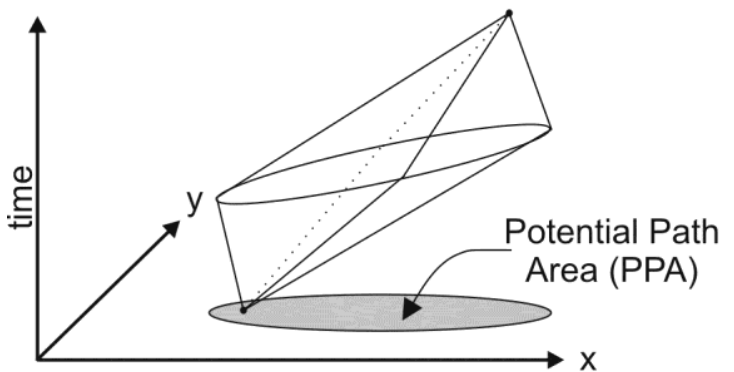

c)

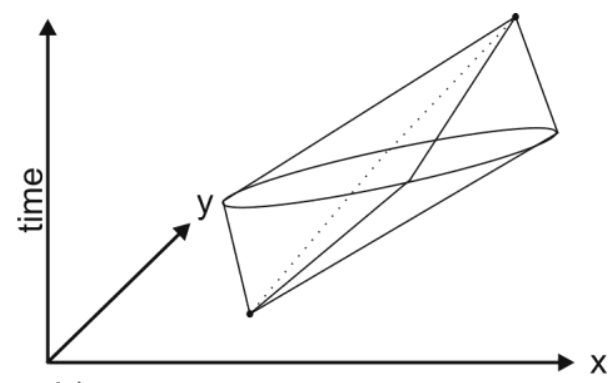

b)

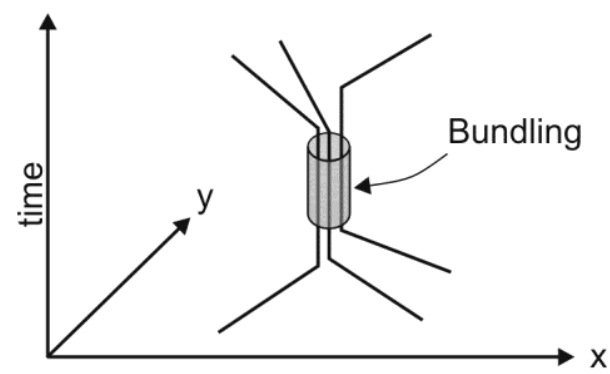

d) 
Figure 2: Comparison between definitions of a) flocks, and b) convoys. A flock requires objects be contained in a circle of radius $-r$, while a convoy is defined as those objects that are density connected at distance $-d$. Both methods require that objects be included in the group over a minimum time interval $-\tau$.

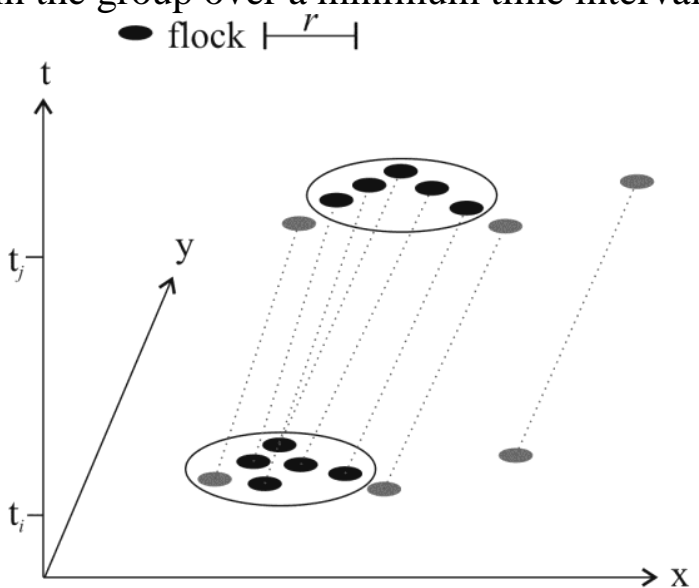

a)

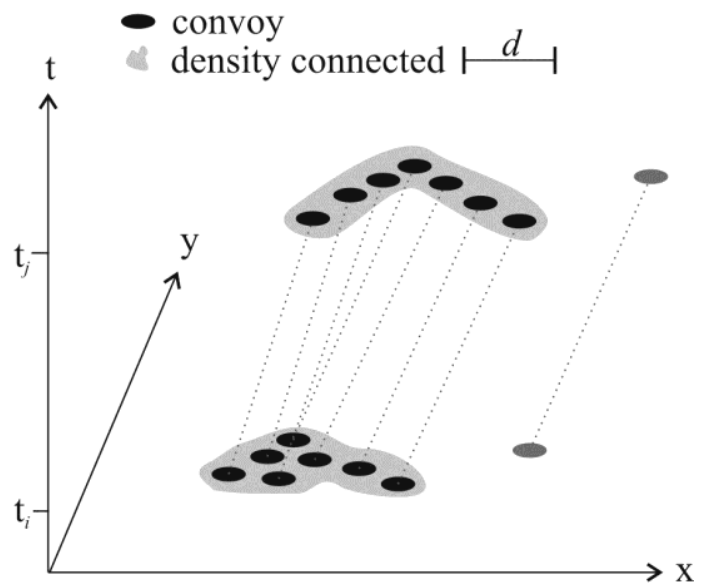

b) 
Figure 3: Four analysis levels for movement data: instantaneous, interval, episodal, and global. After Figure 2 from Laube et al. (2007).

global

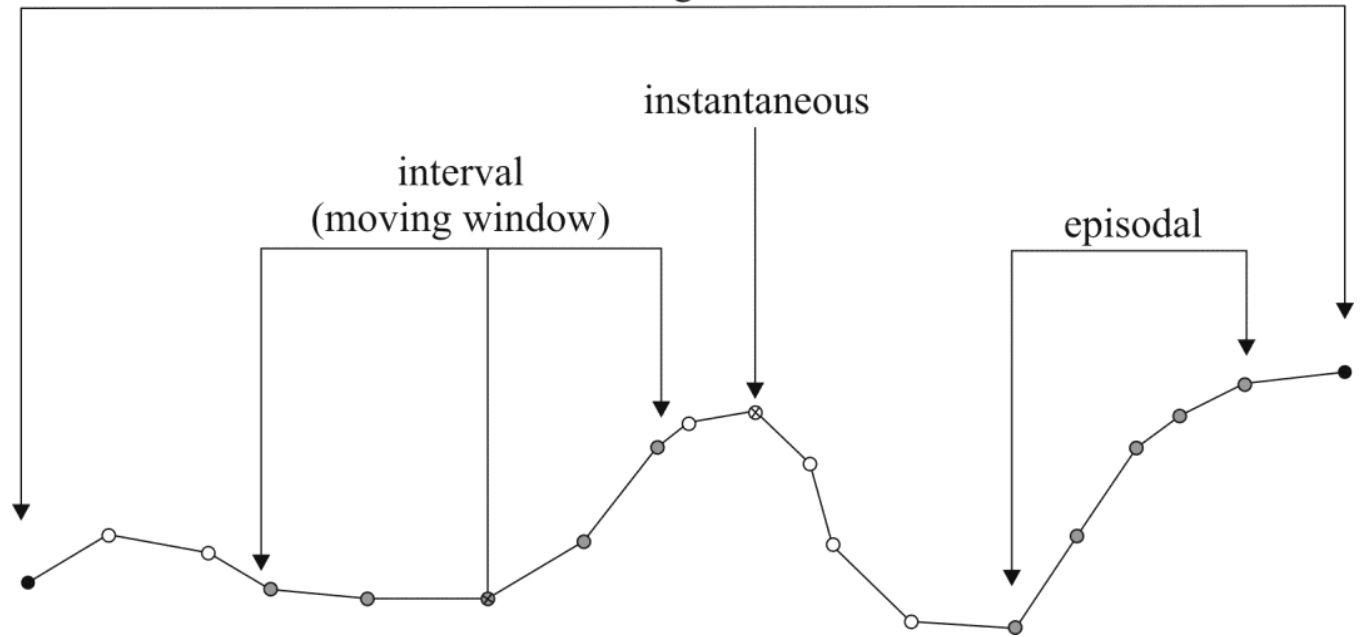

